\title{
A Short Survey: Topological Shape Optimization of Structures Using Level Set Methods
}

\section{Zhen Luo*}

School of Electrical, Mechanical and Mechatronic Systems, The University of Technology, Sydney, NSW 2007, Australia

\begin{abstract}
This paper will give a short survey about topology optimization of structures. It is particularly focused on topological shape optimization of structures using level-set methods, including the level-set based standard methods and the level-set based alternative methods. The former often directly solve the Hamilton-Jacobi partial differential equation (H-J PDE) to obtain the boundary velocity field using Finite Differential Methods (FDM), and the later commonly employ parametric or equivalent methods to evaluate the velocity field without directly solving the H-J PDE. The unique characteristics of the level-set based topology optimization methods are discussed, and a future perspective and prospects in this research area is also included. A benchmark numerical example is used to showcase the effectiveness of the level-set based methods.
\end{abstract}

Keywords: Topology optimization; Level set methods; Structures

\section{Structural Optimization Using Level Set Methods}

Over the past two decades, a relatively new field known as topology optimization is rapidly expanding in computational design research. In contrast to the detailed designs (e.g. size and shape optimizations) of a structure, topology optimization [1] is highly challenging at the conceptual design stage, because it requires automatic determination of an optimal material layout of a structure in conjunction with an optimal shape of the boundary, to make cost-efficient use of a given amount of material for improving the concerned structural performance. Topology optimization can be regarded as an iterative numerical procedure to re-distribute the material in a fixed reference domain subject to boundary conditions. The optimal topology of the structure can be obtained, in association with an optimal material connectivity, when a pre-defined objective function reaches its extremity under specific constraints. Topology optimization has been applied to a broad range of existing research areas [1], and is continuously being introduced to many new and emerging areas, as an enabling persuasive design technique.

Several typical methods have been developed for topology optimization of structures, including the homogenization method [2], the SIMP (Solid Isotropic Material with Penalization) approach $[3,4]$ and the level set-based method [5-8]. Topology optimization essentially belongs to a family of integer programming problems with a large number of discrete design variables. On one hand, many wellestablished more efficient gradient-based optimization algorithms cannot be directly applied, due to the discrete nature of the problem. On the other hand, conventional discrete optimization algorithms, such as the genetic algorithms, may not be used to effectively find the solution of such large-scale discrete optimization problems, due to the "NP-Hard" difficulty. To this end, the homogenization and SIMP are two typical methods, which have been widely used to relax the original discrete optimization problem, to allow the discrete design variables taking intermediate values ranging from 0 to 1 .

In particular, SIMP, as an extension of the homogenization method, has received popularity in the area of structural optimization, due to its conceptual simplicity and implementation easiness. SIMP has already had several variant formulations, including elemental density based SIMP [4], nodal density based SMIP [9] and meshless field points based SIMP [10]. With SIMP, the original discrete optimization is changed to a continuous one in an enlarged design domain. To make the solution close to the original 0 and 1 binary bounds, a 'power-law' criterion [4] is usually applied to SIMP model to penalize intermediate densities, so as to push the design domain to the original one as close as possible. To ensure a physically meaningful solution that is manufacturable in engineering, additional numerical techniques, such as the filtering schemes $[1,11,12]$, are further applied to eliminate checkerboards and mesh-dependence [13-15].

Recently, the level-set based method, originally applied to propagate interfaces [16-18] in fluid mechanics, combustion, computer animation, material science and image processing, is emerging as a new technique well suited to optimizing shape and topology of structures. Sethian and Fedkiw [19] can be regarded as the first a couple of researchers who introduced the level set model into shape and topology optimization of structures. In level set based methods [6-8], the key concept is to implicitly represent the boundary as the zero level-set of a higher dimensional level set function of Lipschitz continuity. The level set function is defined over a fixed reference domain that includes all the admissible shapes and topologies of the design domain.

In level set methods, a pseudo-time is normally introduced into the level set function to enable the dynamic evolution of the discrete level set function $[17,18]$. The level set representation of dynamic implicit surfaces can be mathematically described as a level set equation, namely the Hamilton-Jacobi Partially Differential Equation (H-J PDE). Appropriate numerical schemes [20] are required to obtain the steadystate solution of the H-J PDE. The motion of the level set function along the normal direction will lead to the motion of the design boundary, as well as the shape and topological changes of the structure at the zero level-set. It is noted that the tangent part of the velocity field only

*Corresponding author: Zhen Luo, School of Electrical, Mechanical and Mechatronic Systems, The University of Technology, Sydney, NSW 2007, Australia, Tel: +61-2-9514 2994; Fax: +61-2-9514 2655, E-mail: zhen.luo@uts.edu.au

Received March 23, 2013; Accepted May 17, 2013; Published May 20, 2013

Citation: Luo Z (2013) A Short Survey: Topological Shape Optimization of Structures Using Level Set Methods. J Appl Mech Eng 2: 123. doi:10.4172/21689873.1000123

Copyright: ( $) 2013$ Luo Z. This is an open-access article distributed under the terms of the Creative Commons Attribution License, which permits unrestricted use, distribution, and reproduction in any medium, provided the original author and source are credited. 
contributes to the parameterization of the front $[6,7,19]$.

Commonly, a level-set based topology optimization method should include three core elements:

1. Level-set model [16], to which the design boundary is implicitly represented as the zero level set of a higher dimensional level set function.

2. A system of H-J PDEs $[17,18]$, which can be either directly or indirectly solved using numerical methods, such as up-wind schemes.

3. Shape derivative analysis $[21,22]$ to enable the design sensitivities.

The level-set based method has experienced considerable development in the field of structural optimization. These topological shape optimization methods can be approximately classified into two major categories. The first of which $[6,7,19,23,24]$ is based on the standard level set model $[16,17]$. The major characteristic of these methods is to use Finite Difference Methods (FDM), such as the upwind scheme [6-7], to directly solve the H-J PDE, based on a set of fixed Eulerian grid. The Finite Element Method (FEM) is typically utilized to evaluate the solution (e.g. displacement, strain and stress) of the state equations based on a set of Lagrangian mesh. The normal velocity at the design boundary can be obtained by using shape derivative analysis method. Finally, the velocity field is incorporated into the H-J PDE to enable the update of the discrete level set function values, and then the evolution of the design boundary.

However, in the standard level set methods, to numerically solve a system of complicate PDEs with Finite Difference Methods (FDM) is by no means easy. The numerical difficulties [6-8] related to the CourantFriedrichs-Lewy (CFL) condition, periodically applied re-initializations and velocity extension schemes have to be carefully handled in the numerical process. Furthermore, many efficient gradient-based optimization methods [1] that will be more effective and efficient, such as the OC (optimality criteria) method and SQP (sequential quadratic programming) approach, in the field of design optimization cannot be directly applied to solve these topology optimization problems. These issues have severely limited the application of the level set method to more advanced topology optimization problems.

The CFL condition $[16,17]$ is mainly imposed to ensure numerical stability when the dynamic interface crossing the mesh. So the time step size is strictly restrained by the CFL condition, to ensure the marching size of the boundary is smaller than the minimum grid size in space at each step. In this way, a finer mesh will significantly increase iterations for convergence and as a result the overall computational expense, although a finer mesh may benefit the numerical accuracy to some extent. The re-initializations [6-8] are used to regularize the shape of the level set surface, as the unshaped level set function may lead to too steep or flat regions due to the unwanted dissipation of the front when FDM is used. It is noted [6-8] that the global re-initializations would limit the "nucleation" of the new holes inside the design domain, which will make the final design largely dependent on the initial guess of the design. Hence, to overcome the shortcomings in the first category of the level set methods, the level set model is better when transformed into a parametric or equivalent one to avoid drawbacks of its classic discrete forms, while retain the topological shape benefits of the level set boundary representation. In this way, the level set method will be naturally connected with the more powerful optimization algorithms in the field.

As a result, the second category is the development of alternative level set methods [25-31] for shape and topology optimization of structures, without directly solving the H-J PDE. For instance, Belytscko et al. [29] proposed a "narrow band" method, which represented the level set surface within a range of the zero level set boundaries, based on a set of nodal variables of the level set function. An optimality criteriabased algorithm was employed to update those nodal variables within the narrow band, so as to advance the design boundary. Haber [30] proposed a multilevel continuation scheme, in which SQP was applied to update the implicit shape boundary rather than directly solving the H-J PDE. De Ruiter and Kenlen [31] developed a topological description function method, which only employed the concept of the implicit level set boundary representation to geometrically describe the design boundary, without the consideration of the H-J PDE. Luo et al. [20] proposed a semi-implicit level set method for structural optimization, that is, a semi-implicit additive operator splitting (AOS) scheme rather than the FDM was utilized to solve the H-J PDE numerically. Luo et al. [27] studied a multiphase level set method using the piecewise constant level sets. Luo et al. [25] proposed a powerful level set method using the parameterization of the compactly Supported Radial Basis Function (CSRBF) [25,26]. The discrete level set surface can be uniquely determined in terms of a set of pre-known discrete level set function values at knot positions and their corresponding shape functions. In this way, the complicated H-J PDEs are decoupled into a system of ODEs, and further to algebraic equations. The more difficult topological optimization is therefore transformed into a relatively easier size optimization, to which more efficient gradientbased optimization algorithms can be directly applied. The numerical drawbacks associated with the re-initializations, CFL condition and velocity extension can be reasonably avoided.

Therefore, as discussed above, the level-set topology optimization of structures has developed as a powerful multidisciplinary method, which has been applied to a wide range of scientific and engineering applications across many structural, mechanical and material disciplines [8,24,27,32-37]. In contrast to conventional topology optimization methods (e.g. homogenization and SIMP) that optimize the geometry of a structure by varying densities of a fictitious material, the level set based methods offer the following unique advantages [5-8]

1. Shape fidelity and topological flexibility. The level set method lends itself to a process of topological shape optimization, which indicates a seamless integration of shape optimization and topology optimization in the course of dynamic boundary merging and splitting.

2. Crisp and distinct material interfaces. This often plays an important role for the design problems involving strong interfacial phenomena, while avoiding ambiguities of gray-scale intermediate densities surrounding the design boundary in density-based methods.

3. Smooth boundaries. It is of great importance to problems requiring the description of more accurate response along the interface, while the density-based methods inevitably result in zigzag boundaries, as a result of describing the geometry of the structure using finite elements.

4. A physically meaningful solution can be obtained, based on the theory of viscosity solution of the Hamilton-Jacobi PDEs.

5. Without experiencing checkerboards. The topology optimization design of level sets is free of checkerboards that make a design practically manufacturable.

\section{Numerical Example}

In this section, one benchmark numerical example is conducted to show the effectiveness of the level-set based topology optimization 
methods. It is practically impossible to run all the different level-set methods in this short survey paper. Hence, this research will focus on the parametric level set method [8] for topology optimization of a cantilever-type structure, which has been a well-studied problem widely accepted in the field. It should be good enough to demonstrate the unique characteristics of the level-set based topology optimization methods.

The design domain of the cantilever beam is shown in figure 1 with a size of $\mathrm{L}=50$. The left side is fixed, and a concentrated force $\mathrm{F}=1$ is vertically loaded at the center point of the right side. The design domain is discretized with a mesh of $100 \times 50$ quadrilateral elements. The objective function is to minimize the mean compliance of the structure subject to a volume constraint (e.g. 50\% is used here). The termination criterion for the convergence is the relative difference of the objective function values for two successive iterations is less than 0.01 . The artificial material model is stated as: Young's modulus for the solid material is 1 and for the weak material in the void is 0.0001 to avoid numerical singularity in assembling the system stiffness matrix, and the Poisson's ratio for all material phases is 0.3 . It is noted that it is unnecessary to use explicit units for all the parameters, because the relative elemental densities are used. However, in the whole design optimization, the same set of units is required to be remained.

In the numerical implementation, the strain field of those elements crossed by the level-set boundary can be approximated via the simple but effective "ersatz material" scheme [7]. Although the initial level set surface is a signed distance function, no further re-initializations is applied to keep the shape of the level set surface in the optimization. The shape derivative method $[21,22]$ is applied to find the design sensitivity of the design functions with respect to the design variables. The MMA [36] is used as the optimizer to update the design variables based on the sensitivity information, so as to evolve the level set function and design boundary.

Here, only the linear elastic structure is considered only for numerical simplicity but without losing any generality. The minimum compliance problem can be generally given by

$$
\begin{array}{r}
\underset{\Phi}{\operatorname{Minimize}: J(u, \Phi)}=\int_{D} F(u, v) H(\Phi) d \Omega \\
F(u, v)=\varepsilon_{i j}^{\mathrm{T}}(u) D_{i j k l} \varepsilon_{k l}(v) / 2
\end{array}
$$

Subject to:

$$
\left\{\begin{array}{l}
\int_{D} H(\Phi) d \Omega-V_{0} \leq 0, \\
a(u, v, \Phi)=l(v, \Phi),\left.u\right|_{\Gamma_{D}}=u_{0}, \forall v \in \mathbf{U}
\end{array}\right.
$$

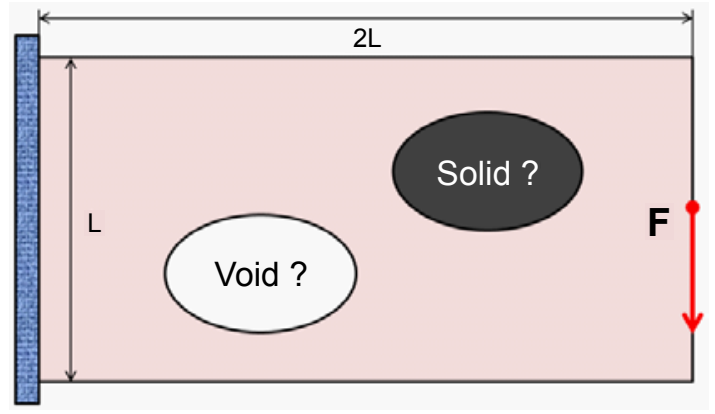

Figure 1: Design domain of a cantilever beam.
Where, the constraint is introduced to limit the material usage, and $V_{0}$ is the prescribed volume ratio. $u$ is the displacement field, $\varepsilon$ is the strain field, $v$ denotes the virtual displacement field belonging to the kinematically admissible $\mathbf{U}$ space, $u_{0}$ is the prescribed displacement on the boundary $\Gamma_{D} . E$ is the material elasticity tensor. $H(\Phi)$ is the Heaviside function to uniformly indicates the different parts (solid, void and boundary) in the reference domain $D[6,7]$.

The state equation $a(u, v, \Phi)=l(v, \Phi)$ is written in its weak variational form in terms of the energy bilinear functional $a(u, v, \Phi)$ and the load linear form $l(v, \Phi)$, which can be defined as

$$
\begin{aligned}
& a(u, v, \Phi)=\int_{D} c(u, v) H(\Phi) d \Omega=\int_{D} \varepsilon_{i j}^{\mathrm{T}}(u) E_{i j k l} \varepsilon_{k l}(v) H(\Phi) d \Omega \\
& l(v, \Phi)=\int_{D} p v H(\Phi) d \Omega+\int_{D} \tau v \delta(\Phi)|\nabla \Phi| d \Omega
\end{aligned}
$$

where $p$ is the body force while $\tau$ is the boundary traction. $\delta(\Phi)$ is the partial derivative of the Heaviside function $H(\Phi)$, namely the Dirac function $[6,7]$.

Figure 2 shows the topological shape designs at different stages, while figure 3 shows their corresponding level set surfaces. From the design results, it can be seen that the proposed level set method is able to flexibly handle structural topology changes and boundary shape fidelity by retaining a smooth boundary and distinct material phases. The topology optimization and shape optimization can be achieved in the same design. The initial level set surface can be processed to implement complex topology and shape evolvements, by merging the exist holes and creating new holes inside the design domain. Furthermore, it can be seen that the numerical difficulties in the standard level set methods, including the CFL condition, periodically applied re-initializations and boundary velocity extension are reasonably avoided [8]. Particularly, the level set models (H-J PDE) are naturally bridged with many wellestablished and more efficient gradient-based optimization algorithms (e.g. OC, SLP, SQP and SCP) in the field of design optimization. These features can greatly benefit the extension and application of the levelset based methods to more advanced problems. It can be found that the parametric level set method owns the unique advantages in the context of level set methods, such as the (1) implicit level-set design boundary representation, (2) level set equation mathematically defined as a firstorder Hamilton-Jacobi PDE, and (3) rigorous shape derivative analysis for the design sensitivities.

Figure 4 displays the convergence of the objective function and constraint over the iterations. The structural mean compliance is minimized from 386.68 to 62.48 after 386 iterations. The volume constraint becomes active after the 8 th iteration $\left(9^{\text {th }}\right.$ and $10^{\text {th }}$ iterations: between $-1.5631 \mathrm{e}-007$ and $1.7545 \mathrm{e}-007)$, and the volume constraint keeps conservative until the optimal design is obtained $\left(385^{\text {th }}\right.$ and $386^{\text {th }}$ iterations: -1.1931e-008 and 1.4187e-008).

\section{Conclusions and Perspectives}

The level-set based method is a recently emerged powerful methodology for shape and topology optimization of structures. The key concept of these methods is to represent the structural boundary implicitly as the zero level set of a higher-dimensional scalar function, which is mathematically defined as a level set equation, and then the shape derivative analysis is included to enable the dynamic motion of the design boundary. A benchmark numerical example has been used to demonstrate the major characteristics of the level-set based methods.

The level-set based methods provide many new features that well suit the optimization of structures. However, the level-set based 


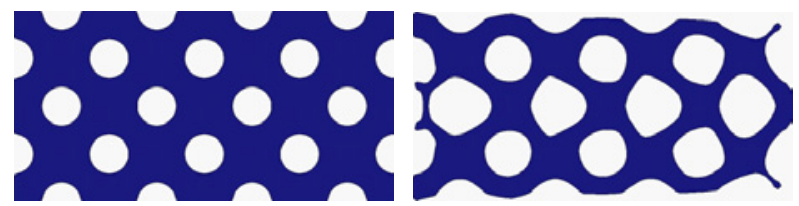

(a) Initial design (step 1)

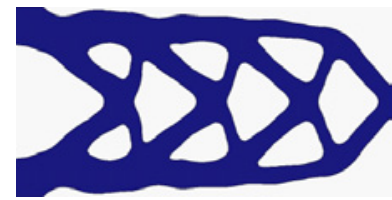

(d) Intermidiate design (step 150) (b) Intermidiate design (step 15)

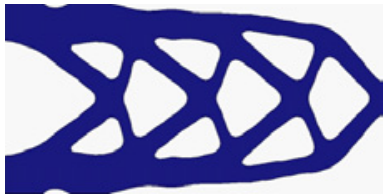

(e) Intermidiate design (step 245)

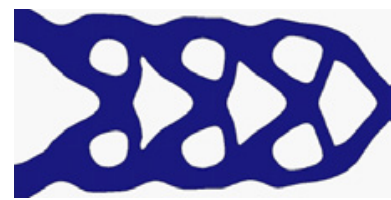

(c) Intermidiate design (step 45)

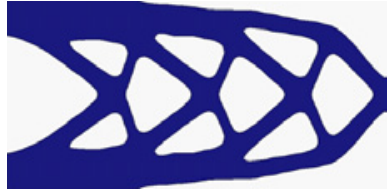

(f) Optimal design (step 386)

Figure 2: Level-set contours of a cantilever beam.

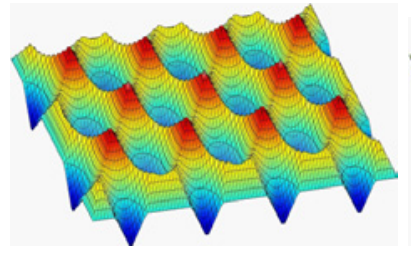

(a) Initial design (step 1)

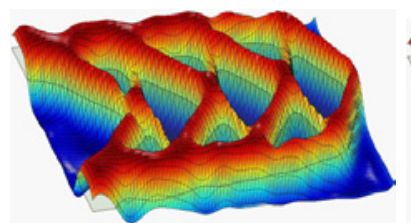

(d) Intermidiate design (step 150)

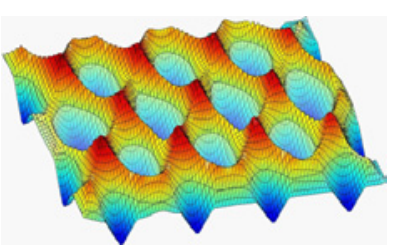

(b) Intermidiate design (step 15)

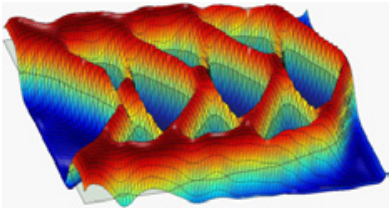

(e) Intermidiate design (step 245)

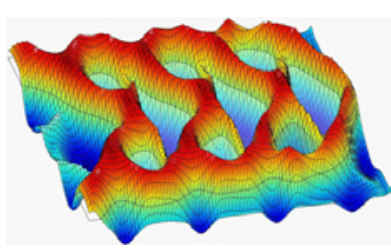

(c) Intermidiate design (step 45)

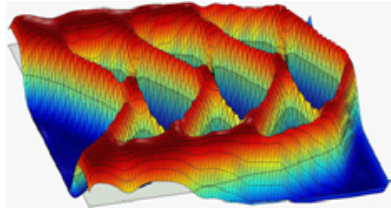

(f) Optimal design (step 386)

Figure 3: Level-set surfaces of a cantilever beam.
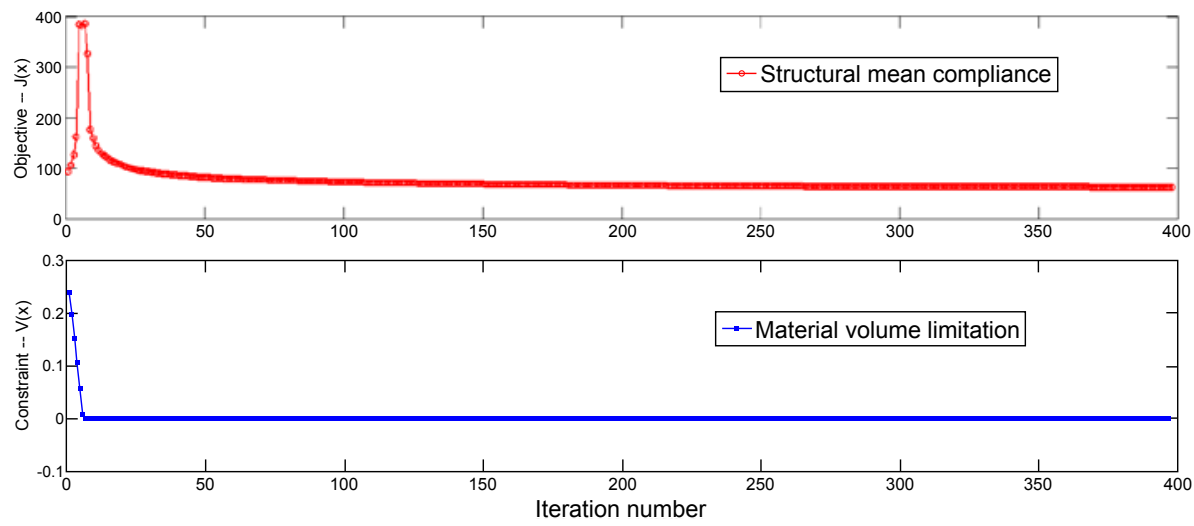

Figure 4: Convergence of the objective function and the volume constraint.

methods will not be limited to the topological shape optimization of structures, and it can be extended to the computational design of mechanical devices and materials in the different fields at macro, micro and nano scales. In particular, the level-set based methods will show their merits in the systematic design of problems at micro- and nano scale, to which a smooth boundary and distinct material phases will play a key role, such as artificially structured meta-materials, structures, mechanical devices including valves and pumps. Hence, the level setbased methods are expected to provide unprecedented opportunities to achieve improved and novel designs in a number of new and emerging areas.

\section{References}

1. Bendsøe MP, Sigmund O (2003) Topology optimization: Theory, Methods, and Applications. Springer, Berlin, Germany.

2. Bendsøe MP, Kikuchi N (1988) Generating optimal topologies in structural design using a homogenization method. Comput Methods Appl Mech Eng 71: 197-224. 
Citation: Luo Z (2013) A Short Survey: Topological Shape Optimization of Structures Using Level Set Methods. J Appl Mech Eng 2: 123. doi:10.4172/2168-9873.1000123

3. Zhou M, Rozvany GIN (1991) The COC algorithm, Part II: topological, geometry and generalized shape optimization. Comput Methods Appl Mech Eng 89: 197 224.

4. Bendsøe MP, Sigmund O (1999) Material interpolation schemes in topology optimization. Arch Appl Mech 69: 635-654.

5. Osher S, Santosa F (2001) Level set methods for optimization problems involving geometry and constraints I. Frequencies of a two-density inhomogeneous drum. J Comput Phys 171: 272-288.

6. Wang MY, Wang XM, Guo DM (2003) A level set method for structural topology optimization. Comput Methods Appl Mech Eng 192: 227-246.

7. Allaire G, Jouve F, Toader AM (2004) Structural optimization using sensitivity analysis and a level-set method. J Comput Phys 194: 363-393.

8. Luo Z, Tong LY, Wang MY, Wang S. (2007) Shape and topology optimization of compliant mechanisms using a parameterization level set method. J Comput Phys 227: 680-705

9. Kang Z, Wang YQ (2012) Structural topology optimization based on non-local Shepard interpolation of density field. Comput Methods Appl Mech Eng 200: 3515-3525.

10. Luo Z, Zhang N, Wang Y, Gao W (2013) Topology optimization of structures using meshless density variable approximants. Int J Numer Methods Eng 93: 443-464.

11. Luo Z, Chen LP, Yang J, Zhang Y, Abdel-Malek K (2005) Compliant mechanism design using multi-objective topology optimization scheme of continuum structures. Structural and Multidisciplinary Optimization 30: 142-154.

12. Luo Z, Chen LP, Yang J, Zhang YQ, Abdel-Malek K (2006) Fuzzy tolerance multilevel approach for structural topology optimization. Computers and structures 84: 127-140.

13. Diaz A, Sigmund O (1995) Checkerboard patterns in layout optimization Structural optimization 10: 40-45.

14. Sigmund O, Petersson J (1998) Numerical instabilities in topology optimization: a survey on procedures dealing with checkerboards, mesh-dependencies and local minima.Structural Optimization 16: 68-75.

15. Luo JZ, Luo Z, Chenc S, Tong L, Wang MY (2008) A new level set method for systematic design of hinge-free compliant mechanisms. Comput Methods Appl Mech Eng 198: 318-331.

16. Osher S, Sethian JA (1988) Front propagating with curvature dependent speed: Algorithms based on Hamilton-Jacobi formulations. J Comput Phys 78: 12-49.

17. Sethian JA (1999) Level set methods and fast marching methods: Evolving interfaces in computational geometry, fluid mechanics, computer version and material science. Cambridge University Press, England, UK.

18. Osher S, Fedkiw RP (2002) Level set methods and dynamic implicit surface. Springer, New York, USA.

19. Sethian JA, Wiegmann A (2000) Structural boundary design via level set and immersed interface methods. J Comput Phys 163: 489-528.

20. Jiang GS, Peng D (2000) Weighted ENO schemes for Hamilton-Jacobi equations, SIAM J Sci Comput 21: 2126-2143.

21. Sokolowski J, Zolesio JP (1992) Introduction to shape optimization: Shape sensitivity analysis. Springer, Berlin, Germany.

22. Choi KK, Kim NH (2005) Structural sensitivity analysis and optimization 1-Linear systems. Springer, New York, USA.

23. Yamada T, Izui K, Nishiwaki S, Takezawa A (2010) A topology optimization method based on the level set method incorporating a fictitious interface energy. Comput Methods Appl Mech Eng 199: 2876-2891.

24. Yamasaki S, Nomura T, Kawamoto A, Sato K, Nishiwaki S (2011) A level setbased topology optimization method targeting metallic waveguide design problems. Int J Numer Methods Eng 87: 844-868.
25. Luo Z, Wang MY, Wang S, Wei P (2008) A level set-based parameterization method for structural shape and topology optimization. Int J Numer Methods Eng 76: 1-26.

26. Luo JZ, Luo Z, Tong LY, Chena L, Tong L, Wang MY (2008) A semi-implicit level set method for structural shape and topology optimization. J Comput Phys 227: 5561-5581

27. Luo Z, Tong LY, Luo JZ, Wei P Wang MY (2009) Design of piezoelectric actuators using a multiphase level set method of piecewise constants. J Comput Phys 28: 2643-2659.

28. Luo Z, Zhang N, Gao W, Ma H (2012) Structural shape and topology optimization using a meshless Galerkin level set method. Int J Numer Methods Eng 90: 369-389.

29. Belytschko T, Xiao S, Parimi C (2003) Topology optimization with implicitly function and regularization. Int J Numer Methods Eng 57: 1177-1196.

30. Haber E (2004) A multilevel, level-set method for optimizing eigenvalues in shape design problems. J Comput Phys 198: 518-534.

31. De Ruiter MJ, Van Keulen F (2004) Topology optimization using a topology description function. Structural Optimization 26: 406-416.

32. Challis VJ, Guest JK (2009) Level set topology optimization of fluids in Stokes flow. Int J Numer Methods Eng 79: 1284-1308.

33. Otomori M, Yamada T, Izui K, Nishiwaki S (2011) Level set-based topology optimisation of a compliant mechanism design using mathematical programming. Mechanical Sciences 2: 91-98.

34. Shim H, Ho VTT, Wang S, Tortorelli DA (2008) Topological shape optimization of electromagnetic problems using level set method and radial basis function. CMES: Comput Model Eng Sci 37: 175-202.

35. Wang MY, Chen S, Wang X, Mei Y (2005) Design of multimaterial compliant mechanisms using level-set methods. J Mech Des 127: 941-956.

36. Luo Z, Zhang N, Ji J-C, Wu T (2012) A meshfree level-set method for topological shape optimization of compliant multiphysics actuators. Comput Methods App Mech Eng 223: 133-152.

37. Svanberg K (1987) The method of moving asymptotes: a new method fo structural optimization. Int J Numer Methods Eng 24: 359-373.

\section{Submit your next manuscript and get advantages of OMICS} Group submissions

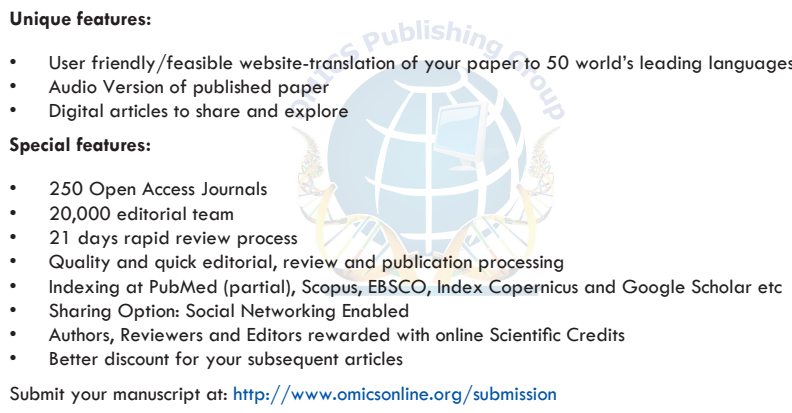

Citation: Luo Z (2013) A Short Survey: Topological Shape Optimization of Structures 